\title{
Análisis de las transiciones entre la formalidad y la informalidad en el mercado de trabajo ecuatoriano
}

\author{
Adriana Patricia Vega Núñez
}

\section{Resumen}

En el estudio se usan datos longitudinales del Ecuador para analizar las transiciones entre el mercado de trabajo formal y el informal. En primer lugar, se emplean matrices de transición para caracterizar la dinámica de corto plazo entre las diferentes situaciones laborales en el Ecuador. A continuación, se utilizan modelos logit multinomiales para averiguar qué factores determinan la probabilidad de permanecer en el sector formal o el informal, o de desplazarse de uno a otro. El nivel de educación, los años de experiencia y las desigualdades salariales entre los sectores tienen un efecto apreciable en las transiciones de los trabajadores, lo que demuestra que los beneficios y los costos varían en función de las preferencias y las habilidades personales.

\section{Palabras clave}

Empleo, mercado de trabajo, sector informal, movilidad de la mano de obra, análisis matemático, estadísticas del empleo, Ecuador

\section{Clasificación JEL}

O170, R230 y J420

\section{Autores}

Adriana Patricia Vega Núñez es Candidata a Doctorado en el Departamento de Econometría, Estadística y Economía Aplicada de la Universidad de Barcelona, España. Correo electrónico: adriana.vega.nunez@gmail.com. 



\section{Introducción}

La informalidad generalizada es una característica de las economías en desarrollo y en transición ${ }^{1}$. En esos países, hay una gran variedad de personas que participan en actividades económicas informales, lo que las afecta de múltiples maneras. Este fenómeno persistente sigue siendo un problema importante en los muchos países que tratan de reducirlo². Charmes (2009) muestra que el empleo informal ha existido desde que se lo definió por primera vez a mediados de los años setenta. Hasta la década de 2000, el empleo informal mostró una tendencia ascendente en varias regiones del mundo ${ }^{3}$. En promedio, en los últimos 30 años, el empleo informal ha representado más del $47 \%$ del total del empleo no agrícola en las regiones en desarrollo, aproximadamente el 24\% en las economías en transición y más del 50\% en América Latina.

Si bien los investigadores definen la informalidad de diferentes maneras, esta suele asociarse con fenómenos negativos: desprotección de los trabajadores, evasión de impuestos, actividades ilegales, baja productividad, bajas tasas de inversión y otros. Sin embargo, en la literatura han surgido distintas escuelas de pensamiento sobre las causas del empleo informal. La pregunta principal es si las personas o las empresas salen del sector formal por voluntad propia o porque se las excluye de él. En la publicación del Banco Mundial, Informalidad: escape y exclusión (Perry y otros, 2007), se examinan dos escuelas dominantes de pensamiento.

Por una parte, se menciona la visión de exclusión, que coincide con el pensamiento tradicional y señala que el mercado se encuentra segmentado y las personas eligen el empleo informal porque es la única alternativa (De Soto, 2000). En ese sentido, los trabajadores del sector informal preferirían empleos formales que ofrecen salarios más elevados y protección laboral.

Por otra parte, se examina el concepto de sector informal voluntario, que se opone diametralmente a la visión anterior porque sugiere que los trabajadores eligen entre los distintos puestos que se ofrecen y se incorporan al sector informal por voluntad propia. Maloney (2004) apoya esta idea y, sobre la base de información de América Latina, sugiere que el sector informal es un sector microempresarial no regulado, en lugar de un residuo desfavorecido de los mercados de trabajo segmentados. Además, Bosch y Maloney (2005) indican que una parte considerable de los trabajadores del sector informal, en particular los que trabajan por cuenta propia, ingresan en ese sector de manera voluntaria. Por consiguiente, hay muchos tipos de empleos informales que pueden ofrecer ventajas deseables, como independencia u oportunidades de capacitación.

El propósito de este estudio es examinar la dinámica del mercado de trabajo en cuanto a las diferentes situaciones laborales y observar las características que determinan la probabilidad de transición o permanencia en el sector formal y el informal en el Ecuador. El análisis empírico se basa en la Encuesta Nacional de Empleo, Desempleo y Subempleo (ENEMDU) del Ecuador, correspondiente al período de 2011 a 2012. Para llevar a cabo el estudio, se examinaron los flujos entre los siguientes sectores laborales: el formal, el informal, el desempleo y el sector de los que están fuera de la fuerza de trabajo. El estudio contribuye a la literatura existente de dos maneras: en primer lugar, la encuesta de panel que se utilizó, en la que se hace un seguimiento de los trabajadores formales e informales durante dos años consecutivos, permite determinar cómo cambia la fuerza de trabajo a lo largo del tiempo; en segundo lugar, en el estudio se aportan datos acerca del Ecuador, país del que se dispone de escasa información. Con ese fin, se creó una matriz de transición para

\footnotetext{
1 Independientemente de los métodos que se usen para medir la informalidad, esta es elevada en América Latina (Perry y otros, 2007). La informalidad ha aumentado con el tiempo en África Subsahariana y Asia (Jütting, Parlevliet y Xenogiani, 2008).

2 Según Baccheta, Ernst y Bustamante (2009), sobre la base definiciones amplias de informalidad, los países de África, Asia y América Latina presentan tasas persistentes de informalidad.

${ }^{3}$ El empleo informal ha ido en aumento en África Subsahariana, Asia Meridional y Oriental, América Latina, Asia Occidental, África Septentrional y los países en transición (Charmes, 2009).
} 
representar la dinámica del mercado y las interacciones entre los sectores. Además, dado que la matriz de transición no refleja las características observables de los trabajadores, se usó un análisis logit multinomial para identificar esas características y averiguar cómo determinan la probabilidad de que se elija un sector laboral determinado.

Este artículo está dividido en cinco secciones. Luego de la presente introducción figura la sección II, en la que se presenta un breve resumen de la literatura empírica sobre la dinámica del mercado de trabajo formal e informal. En la sección III se presentan los datos y las definiciones de informalidad y de otras variables principales, así como los métodos y los modelos econométricos que se utilizaron en el estudio. La sección IV contiene los resultados del estudio. Por último, en la sección $\vee$ se examinan las principales conclusiones y se presentan las observaciones finales.

\section{Análisis de la literatura}

Como se mencionó anteriormente, se utilizan dos perspectivas para explicar la relación entre la informalidad y la segmentación del mercado de trabajo. En la primera se supone que los trabajadores preferirían tener un empleo asalariado formal, pero se ven obligados a incorporarse al sector informal debido a la presencia de restricciones y a la disponibilidad limitada de empleos formales. En el modelo concebido por Harris y Todaro (1970), la fijación de un salario mínimo por encima del salario de equilibrio dio lugar a una limitación de los empleos formales que condujo a la segmentación del mercado de trabajo.

En consonancia con el segundo punto de vista, Maloney (1999) considera que el sector formal y el informal constituyen un mercado integrado en el que los trabajadores eligen entre los diferentes empleos disponibles sobre la base de sus preferencias, habilidades y necesidades. Por lo tanto, los trabajadores que prefieren el empleo informal al formal lo hacen porque el primero ofrece más características deseables.

En esas dos escuelas de pensamiento, se examinan de distintas maneras los diversos flujos del mercado de trabajo y las desigualdades salariales correspondientes entre los sectores. En un mercado segmentado, los flujos que se desplazan del empleo informal al formal deberían superar a los que se desplazan en sentido contrario. En comparación, en un mercado integrado, los flujos entre el empleo formal y el informal deberían desplazarse en ambos sentidos y tener un volumen similar (Fields, 2009).

Los últimos datos indican que en el mercado laboral de los países emergentes hay una considerable movilidad. El desplazamiento de los trabajadores de un empleo a otro, del grupo de los cesantes al de los ocupados y del interior al exterior de la fuerza de trabajo indica la movilidad en un sector. En este sentido, Maloney (1999) utilizó datos de panel de México para analizar las transiciones de los trabajadores entre los sectores y llegó a la conclusión de que las pautas de movilidad en el mercado de trabajo indican que gran parte del sector informal es un destino deseable y que las distintas modalidades de trabajo, es decir, la formal y la informal, están bien integradas. Duryea y otros (2006) examinaron datos sobre los flujos de trabajadores entre los sectores del mercado de trabajo en tres países de América Latina: Argentina, México y la República Bolivariana de Venezuela. Los autores hallaron una movilidad elevada, no solo hacia dentro y hacia fuera del mercado laboral, sino también entre diferentes tipos de empleos. También observaron que, en promedio, los ingresos de los trabajadores que pasaban del empleo asalariado formal al informal disminuían, mientras que la movilidad en sentido inverso producía el efecto contrario. Cea y Contreras (2008) usaron datos de panel para proporcionar información acerca de Chile. Los resultados revelaron una fuerte tendencia de las personas a permanecer en su situación laboral. En el estudio también se sugiere que la edad, 
la instrucción y los ingresos no laborales inciden mucho en la probabilidad de tener una situación laboral determinada. En un estudio sobre la Argentina, Jiménez (2011) encontró pruebas de que el sector formal del mercado de trabajo estaba segmentado. En este caso, un determinado grupo de trabajadores - los asalariados no registrados - permanecía en condiciones laborales desfavorables.

En su análisis de datos de panel de Ucrania, Lehmann y Pignatti (2008) encontraron pruebas de segmentación en el mercado laboral, donde los movimientos de los asalariados informales eran en gran medida involuntarios. Slonimczyk y Gimpelson (2013), utilizando un modelo logit multinomial y considerando la heterogeneidad individual en cuanto a las preferencias, sugirieron que, en la Federación de Rusia, había un mercado de trabajo integrado.

En general, la informalidad es una característica destacada de las economías emergentes y de transición, como también lo es la movilidad de los trabajadores entre los sectores del mercado de trabajo. A lo largo de los años, estos temas han sido objeto de amplias investigaciones en todo el mundo y se han obtenido resultados diversos. No obstante, hay pocos datos sobre el Ecuador, ya que los estudios anteriores del mercado laboral ecuatoriano se han centrado principalmente en la distribución de los salarios y no en los efectos sobre el empleo o las transiciones entre el sector formal y el informal. En un estudio reciente llevado a cabo por Canelas (2014), se examina si los cambios del salario mínimo repercuten sobre las tasas de formalidad e informalidad y sobre los niveles salariales en el Ecuador. Los resultados indican que dichos cambios no afectan las tasas de ocupación ni los salarios.

\section{Datos y metodología}

Los datos que se utilizan en el presente estudio provienen de la Encuesta Nacional de Empleo, Desempleo y Subempleo (ENEMDU), una encuesta rotativa de panel y de hogares que lleva a cabo el Instituto Nacional de Estadística y Censos (INEC) del Ecuador. El panel no se construye sobre la base de un seguimiento continuado de las personas, sino a partir de cuatro informes, distribuidos a lo largo de dos años consecutivos. Se hacen entrevistas a un primer grupo de hogares en dos trimestres consecutivos, luego este se sustituye por una nueva unidad de muestra en los dos trimestres consecutivos siguientes, y después se regresa por última vez a la primera muestra en dos trimestres consecutivos más. En esta encuesta nacional se incluye a las poblaciones urbanas y rurales.

El panel que se analizó va del cuarto trimestre de 2011 al cuarto trimestre de 2012. En el análisis se utilizaron únicamente los datos correspondientes a un año, porque los resultados obtenidos de los cuadros de transición independientes que se calcularon en relación con los años anteriores eran bastante similares a los del panel que se evaluó en este estudio (véanse los cuadros A1.1 y A1.2 del anexo). Los índices de ponderación que se utilizaron reflejan los factores de expansión que indica el INEC. En el análisis se incluyen trabajadores de 15 años y más.

A fin de determinar las distintas situaciones laborales que se utilizaron en el análisis, la fuerza de trabajo se dividió en tres categorías principales: los ocupados, los cesantes y los que se encuentran fuera de la fuerza de trabajo. Además, la categoría de los ocupados se subdividió en dos grupos más: las personas del sector formal y las personas del sector informal, que incluía trabajadores asalariados (en empresas privadas o en el trabajo doméstico) y trabajadores independientes (autoempleo). Así pues, se consideraron cinco situaciones diferentes en el mercado de trabajo: i) empleo asalariado formal, ii) empleo asalariado informal, iii) autoempleo, iv) desempleo y v) fuera de la fuerza de trabajo. Estas divisiones se hicieron en la población en edad de trabajar y eran mutuamente excluyentes. Las personas se clasificaron como cesantes si en la semana de referencia no habían trabajado pero habían buscado trabajo. Se consideró fuera de la fuerza de trabajo a las personas que no estaban trabajando ni buscando ningún tipo de empleo. 
Existen diversas definiciones de informalidad, según diversos criterios, como el tamaño de las unidades productivas o las empresas, o el número de empleados y el tipo de relación laboral. La Organización Internacional del Trabajo (2013) define el sector informal como un conjunto de unidades dedicadas a la producción de bienes o servicios con la finalidad primordial de generar empleo e ingresos para las personas implicadas. Estas unidades suelen funcionar con un bajo nivel de organización y a pequeña escala. Las relaciones laborales se basan sobre todo en el empleo ocasional, el parentesco o las relaciones personales y sociales, y no en arreglos contractuales que supongan garantías formales. En este contexto, y teniendo en cuenta los datos de la encuesta nacional, en el presente estudio se utilizan dos definiciones del sector informal. La primera se refiere a las características de la empresa y abarca el empleo asalariado en pequeños establecimientos no registrados que tienen menos de diez empleados, más todo el trabajo independiente y por cuenta propia. La segunda se basa en la cobertura de seguridad social: se considera que los trabajadores están en el sector informal si no cuentan con dicha cobertura.

En el gráfico 1 se ilustra la tasa de informalidad en el período de observación de 2011 a 2012 a partir de las dos definiciones mencionadas. Como se puede apreciar en el gráfico, la proporción de trabajadores informales en la fuerza de trabajo del Ecuador era de entre el 32\% y el 38\% en el período de observación. En general, si bien la tasa de informalidad del Ecuador puede considerarse alta, se encuentra dentro del promedio correspondiente al empleo informal en la mayoría de los países de América Latina ${ }^{4}$.

Gráfico 1

Ecuador: proporción de trabajadores informales en el total de la fuerza de trabajo, 2011-2012 (En porcentajes)

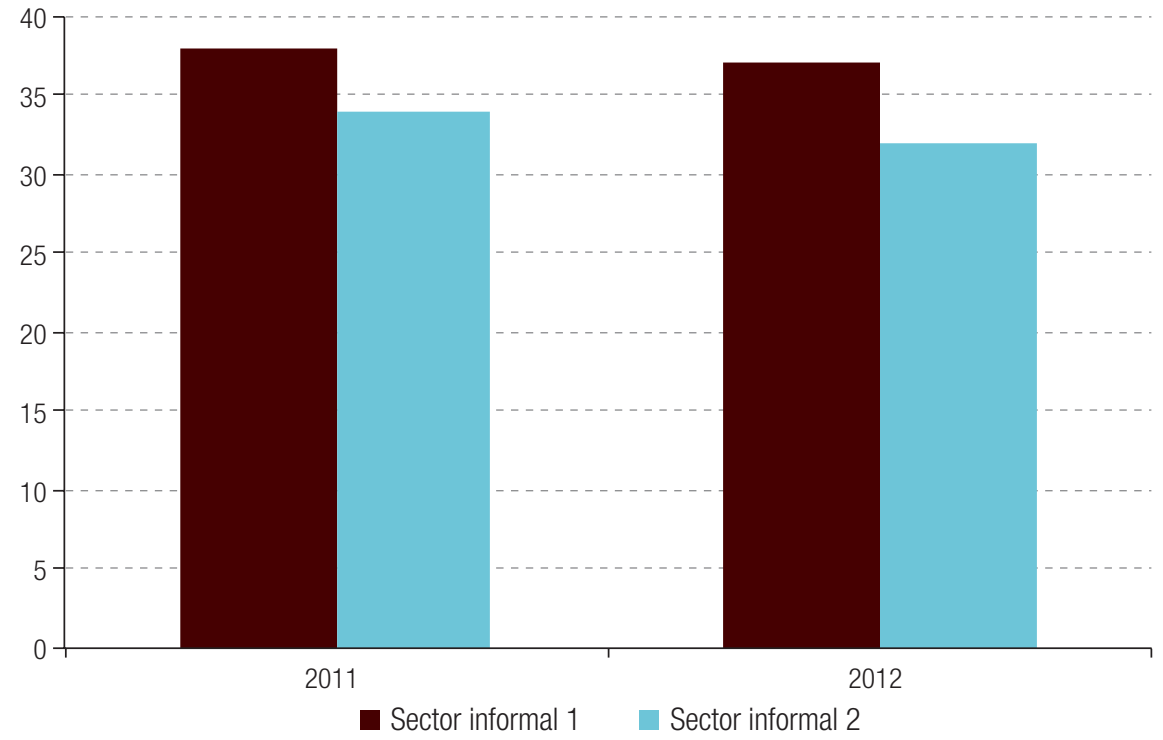

Fuente: Cálculos propios, sobre la base de la Encuesta Nacional de Empleo, Desempleo y Subempleo (ENEMDU) del Ecuador, 2011-2012.

Nota: El sector informal 1 se compone de trabajadores asalariados empleados en pequeños establecimientos no registrados que tienen menos de diez empleados, más todos los trabajadores independientes y los que trabajan por cuenta propia. El sector informal 2 se compone de trabajadores que no tienen cobertura de seguridad social.

\footnotetext{
4 Entre la década de 1970 y la de 2000, el empleo informal representaba en promedio más del $47 \%$ del total del empleo no agrícola en Asia Occidental y África Septentrional, más del 70\% en África Subsahariana, más del 50\% en América Latina, casi el 70\% en Asia Meridional y Sudoriental, y el 24\% en las economías en transición (Charmes, 2009).
} 
Teniendo en cuenta las cinco situaciones laborales, se establecieron 20 transiciones diferentes para representar los flujos entre los sectores del mercado de trabajo durante el período analizado. También se incluyeron cinco estados de permanencia que indican la ausencia de cambios en la situación laboral con respecto al período anterior. El análisis se centra sobre todo en los movimientos desde el sector formal y el informal (empleo asalariado y autoempleo) hacia cada una de las demás situaciones laborales, a fin de determinar las características de los trabajadores que llevan a cabo esas transiciones. En el cuadro A1.3 del anexo se muestran los valores de la media de la muestra, sobre la base de la primera definición de informalidad, en relación con la edad, el nivel de instrucción, la experiencia laboral y el salario real inicial, respectivamente, de los trabajadores que pasan de un sector a otro o que permanecen en su sector. El salario real inicial y el final denotan el salario real mensual de los trabajadores en diciembre de 2011 y diciembre de 2012, respectivamente (considerando los ingresos que provienen del empleo principal como la única fuente de ingresos laborales). Por último, la diferencia entre los ingresos corresponde a la diferencia entre el salario real final y el inicial de los trabajadores que se desplazan a un sector laboral o permanecen en él.

En el resto de esta sección, se describen las técnicas econométricas que se utilizaron en el estudio para proporcionar datos empíricos sobre las pautas de movilidad en los sectores mencionados del mercado laboral ecuatoriano. Para determinar el sentido y el volumen de los diversos flujos del mercado de trabajo, se utilizaron dos métodos: matrices de transición y un análisis logit multinomial de los movimientos entre los sectores.

\section{Matrices de transición}

Las matrices de transición permiten determinar el flujo de trabajadores entre los sectores laborales seleccionados mediante el cálculo de la probabilidad condicionada de encontrar un trabajador en el estado $j$ a finales del período, si, al inicio, el trabajador se encontraba en el estado $i$. Dicha probabilidad se denota con $P_{i j}$. El resultado de la suma de cada fila de la matriz de transición equivale al 100\% y los totales al final de las columnas y las filas representan la proporción de trabajadores que se encuentran en cada categoría al concluir el período $P_{i}$ y $P_{j}$. Los componentes de la diagonal principal revelan la proporción de trabajadores que permanecieron en la misma categoría laboral al final del período. La información que proporcionan las matrices de transición nos da una primera idea de los diferentes movimientos de los trabajadores entre los sectores.

\section{Análisis logit multinomial de las transiciones entre los sectores laborales}

Dado que el método anterior es meramente descriptivo, también se utilizó un análisis logit multinomial para determinar qué características afectan la probabilidad de que los trabajadores opten por pasar al sector $j$ en relación con la probabilidad de permanecer en el sector $i$. El modelo logit multinomial es un modelo probabilístico discreto que permite explicar la tendencia a desplazarse hacia los diferentes sectores del mercado de trabajo o a permanecer en ellos. Permite hallar el efecto que cada característica de los trabajadores tiene sobre la probabilidad de que se desplacen hacia un sector. Se elaboró un modelo de los flujos entre cinco situaciones diferentes del mercado de trabajo: empleo asalariado formal $(j=1)$, empleo asalariado informal $(j=2)$, autoempleo $(j=3)$, desempleo $(j=4)$ y fuera de la fuerza de trabajo $(j=5)$.

Se utilizó la forma exponencial estándar del análisis logit multinomial:

$$
P_{i j}=\frac{\exp \left(X_{i} \beta_{j}\right)}{\sum_{l=1}^{m} \exp \left(X_{i} \beta_{j}\right)}
$$


donde el vector $\beta_{j}$ mide el grado en que un aumento de la característica $X_{i}$ del trabajador incrementa la probabilidad de que este se desplace al estado $j$ en relación con la probabilidad de que permanezca en el estado $i$. Las características del trabajador son la edad, el sexo, el estado civil, el nivel de instrucción, los años de experiencia, la región y los logaritmos de la diferencia entre los ingresos. Se puede calcular la probabilidad de transición donde las variables explicativas determinan el aumento o la disminución de las probabilidades.

\section{Resultados}

\section{Matrices de transición}

En el primer análisis, que se lleva a cabo con matrices de transición, se describe la movilidad de la mano de obra calculando la probabilidad condicionada de que, al final del período, un trabajador se encuentre en el estado $j$, si al inicio se encontraba en el estado $i$. Por lo tanto, se obtiene una matriz anual de 5x5 correspondiente al período analizado, que va de diciembre de 2011 a diciembre de 2012 (véase el cuadro 1). Dado que el objeto del presente estudio es determinar las pautas de las transiciones de los trabajadores hacia fuera y hacia dentro del sector formal y el informal, nos centramos sobre todo en la transición desde el sector formal hacia cada una de las otras situaciones laborales y desde el sector informal (empleo asalariado y autoempleo) hacia los demás sectores. La matriz también revela algunos datos relativos a la dinámica del mercado y a las relaciones entre los sectores definidos.

\section{Cuadro 1}

Ecuador: movilidad de los trabajadores entre los sectores del mercado de trabajo, 2011-2012 (En porcentajes)

\begin{tabular}{|c|c|c|c|c|c|c|c|}
\hline \multirow[b]{2}{*}{ Situación inicial } & \multicolumn{6}{|c|}{ Situación final } & \multirow[b]{2}{*}{$P_{i}$} \\
\hline & $\begin{array}{l}\text { Empleo } \\
\text { asalariado } \\
\text { formal }\end{array}$ & $\begin{array}{c}\text { Empleo } \\
\text { asalariado } \\
\text { informal }\end{array}$ & $\begin{array}{l}\text { Autoempleo } \\
\text { informal }\end{array}$ & Desempleo & $\begin{array}{l}\text { Fuera de la } \\
\text { fuerza de } \\
\text { trabajo }\end{array}$ & Total & \\
\hline Empleo asalariado formal & 79 & 6 & 6 & 2 & 6 & 100 & 25 \\
\hline Empleo asalariado informal & 21 & 46 & 18 & 4 & 12 & 100 & 10 \\
\hline Autoempleo informal & 8 & 7 & 67 & 1 & 16 & 100 & 25 \\
\hline Desempleo & 23 & 11 & 25 & 19 & 22 & 100 & 4 \\
\hline Fuera de la fuerza de trabajo & 4 & 4 & 8 & 3 & 81 & 100 & 36 \\
\hline$P_{j}$ & 26 & 10 & 24 & 3 & 37 & & \\
\hline
\end{tabular}

Fuente: Elaboración propia, sobre la base de la Encuesta Nacional de Empleo, Desempleo y Subempleo (ENEMDU) 2011-2012. Nota: $\quad P_{i}$ es el tamaño relativo de un sector en el período inicial; $P_{j}$ es el tamaño relativo de un sector en el período final.

En lo que respecta al movimiento de los trabajadores que al inicio se encontraban en el sector formal, se halló una tasa de permanencia del 79\%. Es interesante observar que la mayor parte de las personas que salen de este sector lo hacen hacia la categoría informal (12\% en total), en dos flujos distintos. El primero es el movimiento hacia el sector asalariado informal (6\%) y revela el deterioro de las condiciones laborales de los trabajadores en lo que respecta a la formalidad. Este tipo de transición laboral particular podría explicarse por el racionamiento de las oportunidades de obtener un empleo formal (Fields, 1972; Perry y otros, 2007). El segundo es el movimiento hacia el autoempleo informal (6\%), que puede estar relacionado con una decisión voluntaria de las personas que tratan de obtener una mayor autonomía, o puede ser el resultado de la ineficiencia de las protecciones que se ofrecen en el sector formal y del bajo nivel de productividad laboral (Maloney, 1999). Además, los trabajadores del sector formal que abandonan sus puestos de trabajo también pueden pasar al desempleo (2\%) o quedar fuera de la fuerza de trabajo (6\%). 
Los movimientos de los trabajadores asalariados informales presentan un panorama diferente. En primer lugar, la probabilidad de que permanezcan en este sector es de alrededor del $46 \%$, lo que significa que esas personas prefieren buscar otras oportunidades de empleo en lugar de permanecer en estos puestos de trabajo. Si se examinan los flujos de salida de este segmento del sector informal, se observa que el $21 \%$ de los trabajadores obtuvo un empleo de mejor calidad al pasarse al sector formal. Este tipo de movilidad podría interpretarse como una mejora de las condiciones laborales de los trabajadores, dado que los del sector informal suelen ganar menos que sus similares del sector formal (Günther y Launov, 2006). La otra subdivisión del sector informal representa alrededor del $18 \%$ de las salidas, lo que pone de relieve el hecho de que los trabajadores asalariados del sector informal no solo buscan trabajo formal, sino que también procuran trabajar por cuenta propia. Hay características del sector formal y del autoempleo - en particular, en el primer caso, las condiciones laborales y, en el segundo, la independencia- que los hacen algo más atractivos que el empleo asalariado informal. Por lo tanto, la probabilidad de pasar del empleo asalariado informal al formal o al autoempleo es mayor que la probabilidad de pasar al desempleo. El flujo desde el empleo asalariado informal hacia fuera de la fuerza de trabajo es del $12 \%$.

La tercera línea del cuadro 1 ilustra las transiciones desde el autoempleo hacia los demás sectores. Una primera observación es que la tasa de permanencia en este sector muestra que una proporción considerable de personas siguen trabajando por cuenta propia. Una segunda es que los flujos de trabajadores que dejan el autoempleo para ingresar al sector asalariado formal y al informal son bastante similares (8\% y $7 \%$, respectivamente), mientras que la transición hacia el desempleo es del 1\%. Mandelman y Montes-Rojas (2009) sugieren dos motivos posibles que explican la transición hacia el autoempleo: el sector atrae a las personas con capacidades empresariales sobresalientes o constituye un refugio para los cesantes. Cualquiera de estos motivos podría explicar la considerable tasa de permanencia en este sector y la similitud de los flujos hacia el sector formal y hacia el asalariado informal.

Se observó una gran movilidad hacia fuera del sector del desempleo, ya que solo el 19\% de las personas permanecieron en esa situación durante el período de observación. La mayoría de los cesantes se desplazó hacia el autoempleo, seguido por el sector formal y el del empleo asalariado informal, en proporciones del $25 \%$, el $23 \%$ y el $11 \%$, respectivamente.

La mayoría de las personas que encontraron trabajo lo hicieron en el sector informal (empleo asalariado y autoempleo), lo que puede atribuirse a la falta de capital humano o a otras características o preferencias de los trabajadores individuales. En la visión tradicional que considera el desempleo como la condición inferior del mercado laboral, las personas que se encuentran en esa situación pueden tener dificultades para ingresar en el sector formal y, por lo tanto, ser más propensos a aceptar un empleo que ofrece condiciones laborales inferiores y menores ingresos. Jütting, Parlevliet y Xenogiani (2008) sostienen que también es posible que trabajar en el sector informal ofrezca más ventajas que trabajar en el sector formal. Después de todo, la informalidad no puede considerarse el último recurso del trabajador. Es posible que los trabajadores elijan de forma voluntaria el sector informal, donde podrían tener la oportunidad de acumular experiencia o capacitación - sobre todo en el caso de los trabajadores jóvenes poco calificados o los mayores sin calificación- o encontrar mayor flexibilidad y autonomía. El Banco Mundial (2012) destaca que los emprendedores valoran la independencia y la flexibilidad que brinda el contar con una empresa propia y pueden considerar la autonomía como el motivo principal para hacerlo.

Al examinar el sector que está fuera de la fuerza de trabajo se observa una tasa elevada de permanencia. Las transiciones hacia las categorías del autoempleo, el sector formal, el sector asalariado informal y el desempleo representaron solo el $8 \%$, el 4\%, el 4\% y el 3\%, respectivamente. Una vez más, el total de las salidas hacia el sector informal es superior al de las salidas hacia el sector formal. En este contexto, es evidente que el sector informal tiene algunas características atractivas para los trabajadores. 


\section{Análisis logit multinomial}

El objetivo principal de esta sección es obtener un panorama general de la dinámica de la fuerza de trabajo en el Ecuador. Es así que el sector informal, que comprende asalariados y trabajadores por cuenta propia, es la mayor fuente de empleo. Como se mencionó con anterioridad, este segmento de la fuerza de trabajo no necesariamente se corresponde con una situación laboral inferior a la que ofrece el sector formal, habida cuenta de la gran cantidad de trabajadores que pasan de este último al primero.

El análisis logit multinomial del movimiento entre los sectores de trabajo permite determinar, en términos estadísticos, la probabilidad de que los trabajadores pasen del sector inicial a otro sector, sobre la base de sus características específicas (véanse los cuadros 2 y 3).

\section{Cuadro 2}

Ecuador: análisis logit multinomial de las transiciones hacia fuera del sector formal, 2011-2012 (Coeficientes estimados)

\begin{tabular}{|c|c|c|c|c|}
\hline \multirow[b]{2}{*}{ Variables } & \multicolumn{4}{|c|}{ Desde el sector formal hacia } \\
\hline & $\begin{array}{l}\text { Empleo asalariado } \\
\text { informal }\end{array}$ & $\begin{array}{c}\text { Autoempleo } \\
\text { informal }\end{array}$ & Desempleo & $\begin{array}{c}\text { Fuera de la fuerza } \\
\text { de trabajo }\end{array}$ \\
\hline \multirow{2}{*}{ Constante } & 1,391 & $-2,305$ & $-3,387$ & $-1,548$ \\
\hline & $(3,15)^{\star \star \star}$ & $(5,07)^{\star \star \star}$ & $(2,90)^{\star \star \star}$ & $(2,48)^{\star *}$ \\
\hline \multirow{2}{*}{ Edad } & $-0,022$ & 0,026 & $-0,018$ & 0,006 \\
\hline & $(2,56)^{\star *}$ & $(3,52)^{\star \star \star}$ & $(0,90)$ & $(0,52)$ \\
\hline \multirow{2}{*}{ Hombre $^{a}$} & $-0,144$ & 0,026 & $-0,014$ & 0,811 \\
\hline & $(0,78)$ & $(0,15)$ & $(0,04)$ & $(3,42)^{\star \star \star *}$ \\
\hline \multirow{2}{*}{ Casado $^{\mathrm{a}}$} & $-0,365$ & 0,206 & $-1,003$ & $-0,469$ \\
\hline & $(1,93)^{*}$ & $(1,20)$ & $(1,89)^{\star *}$ & $(1,72)^{*}$ \\
\hline \multirow{2}{*}{ Instrucción } & $-0,397$ & $-0,153$ & $-0,277$ & $-0,441$ \\
\hline & $(8,93)^{\star \star \star}$ & $(3,69)^{\star \star \star}$ & $(2,66)^{\star \star \star}$ & $(7,02)^{\star \star \star \star}$ \\
\hline \multirow{2}{*}{ Experiencia } & $-0,005$ & $-0,042$ & $-0,077$ & $-0,008$ \\
\hline & $(0,40)$ & $(4,11)^{\star \star \star}$ & $(1,93)^{\star \star}$ & $(0,56)$ \\
\hline \multirow{2}{*}{ Costa } & $-0,050$ & 0,204 & 1,522 & $-0,436$ \\
\hline & $(0,23)$ & $(0,86)$ & $(1,97)^{\star \star \star}$ & $(1,42)$ \\
\hline \multirow{2}{*}{ Centro } & $-0,069$ & 0,388 & 1,503 & $-0,618$ \\
\hline & $(0,22)$ & $(1,34)$ & $(1,68)^{\star \star}$ & $(1,51)$ \\
\hline \multirow{2}{*}{ Sur } & $-0,257$ & 0,318 & 1,221 & 0,227 \\
\hline & $(0,87)$ & $(1,13)$ & $(1,33)$ & $(0,59)$ \\
\hline \multirow{2}{*}{ Log. dif. de ingresos } & $-0,017$ & $-0,176$ & $-0,778$ & $-0,887$ \\
\hline & $(0,30)$ & $(4,13)^{\star \star \star}$ & $(10,17)^{\star \star \star}$ & $(17,91)^{\star \star \star}$ \\
\hline
\end{tabular}

Fuente: Elaboración propia, sobre la base de la Encuesta Nacional de Empleo, Desempleo y Subempleo (ENEMDU) del Ecuador, 2011-2012.

Nota: Los coeficientes reflejan la manera en que las diferentes características de los trabajadores y la variación porcentual del salario real afectan la probabilidad de pasar del sector inicial al final, en relación con la probabilidad de permanecer en el sector inicial. El sector informal se compone de trabajadores asalariados empleados en pequeños establecimientos no registrados que tienen menos de diez empleados, más todos los trabajadores independientes y los que trabajan por cuenta propia. Las estadísticas Z figuran entre paréntesis; ${ }^{*} p<0,1,{ }^{* \star} p<0,05,{ }^{\star * *} p<0,01$.

a Estas son variables ficticias; las categorías predeterminadas son las siguientes: hombres, estado civil excluidos los casados y región del Norte. 


\section{Cuadro 3}

Ecuador: análisis logit multinomial de las transiciones hacia fuera del sector informal, 20112012

(Coeficientes estimados)

\begin{tabular}{|c|c|c|c|c|c|c|c|c|}
\hline \multirow[b]{2}{*}{ Variables } & \multicolumn{4}{|c|}{ Desde el empleo asalariado informal hacia } & \multicolumn{4}{|c|}{ Desde el autoempleo informal hacia } \\
\hline & $\begin{array}{l}\text { Empleo } \\
\text { asalariado } \\
\text { formal }\end{array}$ & $\begin{array}{c}\text { Autoempleo } \\
\text { informal }\end{array}$ & Desempleo & $\begin{array}{c}\text { Fuera de } \\
\text { la fuerza } \\
\text { de trabajo }\end{array}$ & $\begin{array}{l}\text { Empleo } \\
\text { asalariado } \\
\text { formal }\end{array}$ & $\begin{array}{c}\text { Empleo } \\
\text { asalariado } \\
\text { informal }\end{array}$ & Desempleo & $\begin{array}{l}\text { Fuera de } \\
\text { la fuerza } \\
\text { de trabajo }\end{array}$ \\
\hline \multirow{2}{*}{ Constante } & $-1,774$ & $-3,022$ & $-5,078$ & $-5,392$ & $-3,084$ & 0,404 & $-3,691$ & $-2,908$ \\
\hline & $(3,50)^{\star \star \star}$ & $(5,65)^{\star \star \star}$ & $(4,21)^{\star \star \star}$ & $(5,37)^{\star \star \star}$ & $(6,61)^{\star \star \star *}$ & $(0,88)$ & $(3,17)^{\star \star \star}$ & $(6,04)^{\star \star \star}$ \\
\hline \multirow{2}{*}{ Edad } & $-0,004$ & 0,034 & 0,004 & 0,024 & $-0,016$ & $-0,027$ & $-0,061$ & 0,004 \\
\hline & $(0,43)$ & $(4,13)^{\star \star \star}$ & $(0,23)$ & $(1,63)$ & $(2,15)^{\star \star}$ & $(3,95)^{\star \star \star}$ & $(3,21)^{\star \star \star}$ & $(0,62)$ \\
\hline \multirow{2}{*}{ Hombre $^{\mathrm{a}}$} & $-0,334$ & $-0,154$ & $-0,594$ & 0,744 & $-0,436$ & $-0,589$ & $-0,346$ & 1,078 \\
\hline & $(1,68)^{\star \star}$ & $(0,77)$ & $(1,28)$ & $(2,07)^{\star \star}$ & $(2,67)^{\star \star \star}$ & $(3,52)^{\star \star \star}$ & $(0,87)$ & $(6,44)^{\star \star \star}$ \\
\hline \multirow{2}{*}{ Casado $^{\mathrm{a}}$} & 0,063 & 0,022 & $-0,848$ & $-0,932$ & 0,464 & $-0,411$ & $-0,984$ & $-0,391$ \\
\hline & $(0,29)$ & $(0,11)$ & $(1,58)$ & $(2,30)^{\star \star}$ & $(2,70)^{\star \star \star}$ & $(2,40)^{\star \star}$ & $(1,95)^{\star \star}$ & $(2,45)^{\star \star}$ \\
\hline \multirow{2}{*}{ Instrucción } & 0,295 & 0,157 & 0,246 & 0,168 & 0,303 & $-0,150$ & 0,319 & $-0,064$ \\
\hline & $(5,21)^{\star \star \star}$ & $(2,73)^{\star \star \star}$ & $(1,91)^{\star \star}$ & $(1,63)$ & $(7,35)^{\star \star \star}$ & $(3,23)^{\star \star \star}$ & $(2,92)^{\star \star \star}$ & $(1,49)$ \\
\hline \multirow{2}{*}{ Experiencia } & $-0,018$ & $-0,005$ & 0,004 & $-0,011$ & $-0,005$ & $-0,001$ & $-0,104$ & $-0,017$ \\
\hline & $(1,47)$ & $(0,48)$ & $(0,19)$ & $(0,59)$ & $(0,53)$ & $(0,15)$ & $(02,48)^{\star *}$ & $(2,41)^{\star \star}$ \\
\hline \multirow{2}{*}{ Costa } & $-0,565$ & 0,187 & 0,135 & $-0,037$ & $-0,332$ & $-0,165$ & 0,577 & 0,430 \\
\hline & $(2,39)^{\star *}$ & $(0,75)$ & $(0,24)$ & $(0,08)$ & $(1,52)^{\star \star}$ & $(0,75)$ & $(0,87)$ & $(1,93)^{\star \star}$ \\
\hline \multirow{2}{*}{ Centro } & 0,095 & $-0,086$ & 0,334 & $-0,270$ & $-0,040$ & $-0,168$ & 0,325 & $-0,164$ \\
\hline & $(0,32)$ & $(0,26)$ & $(0,47)$ & $(0,47)$ & $(0,15)$ & $(0,59)$ & $(0,37)$ & $(0,56)$ \\
\hline \multirow{2}{*}{ Sur } & $-0,499$ & $-0,466$ & 0,167 & 0,099 & $-0,132$ & $-0,110$ & 1,318 & 0,406 \\
\hline & $(1,64)$ & $(1,33)$ & $(0,22)$ & $(0,16)$ & $(0,50)$ & $(0,39)$ & $(1,74)^{\star \star}$ & $(1,45)$ \\
\hline \multirow{2}{*}{$\begin{array}{l}\text { Log. dif. de } \\
\text { ingresos }\end{array}$} & 0,034 & $-0,283$ & $-0,942$ & $-1,146$ & 0,126 & 0,174 & $-0,636$ & $-0,661$ \\
\hline & $(0,44)$ & $(4,28)^{\star \star \star}$ & $(9,89)^{\star \star \star}$ & $(13,22)^{\star \star \star}$ & $(3,42)^{\star \star \star}$ & $(4,38)^{\star \star \star}$ & $(7,69)^{\star \star \star}$ & $(19,63)^{\star \star \star}$ \\
\hline
\end{tabular}

Fuente: Elaboración propia, sobre la base de la Encuesta Nacional de Empleo, Desempleo y Subempleo (ENEMDU) $2011-2012$.

Nota: Los coeficientes reflejan la manera en que las diferentes características de los trabajadores y la variación porcentual del salario real afectan la probabilidad de pasar del sector inicial al final, en relación con la probabilidad de permanecer en el sector inicial. El sector informal se compone de trabajadores asalariados empleados en pequeños establecimientos no registrados que tienen menos de diez empleados, más todos los trabajadores independientes y los que trabajan por cuenta propia. Las estadísticas Z figuran entre paréntesis; ${ }^{*} p<0,1,{ }^{* *} p<0,05,{ }^{* * *} p<0,01$.

a Variables ficticias: las categorías predeterminadas son las siguientes: hombres, estado civil excluidos los casados y región del Norte.

Los resultados obtenidos indican que los trabajadores tienen menos probabilidades de pasar de la situación formal a la informal a medida que aumenta su nivel de educación. En promedio, los trabajadores que pasaron al empleo asalariado informal y al autoempleo habían finalizado seis y siete años de estudios, respectivamente.

En cuanto a la experiencia, la probabilidad de pasar al sector informal, en particular al autoempleo, disminuye a medida que aumenta la experiencia del trabajador. Cabe señalar que ocho es la media de años de experiencia de las personas que establecieron sus propias empresas, lo que representa una cantidad considerable de años de capacitación. Aroca y Maloney (1998) hallaron que el sector del autoempleo informal es un destino deseable para los trabajadores, pero requiere una acumulación de capital financiero y humano. Así pues, la media de años de experiencia indica que los trabajadores primero acumulan ahorros y conocimientos, que luego pueden utilizarse para crear una empresa. Las características de cada uno de los sectores del mercado laboral y las propias de cada empleo determinan las diferencias salariales entre los sectores. Tansel y Oznur (2012) examinaron si los trabajadores informales recibían remuneraciones inferiores a las de los trabajadores similares del sector formal. Los autores concluyeron que había efectos fijos no observados que, combinados con las características observables de los trabajadores, explicaban las diferencias de remuneración entre el empleo formal y el informal. 
Por lo tanto, las características no observadas, como la capacidad de producción personal, los rasgos del carácter y la calidad de la gestión, podrían afectar la productividad de los trabajadores y, por consiguiente, las desigualdades salariales. Como era de esperar, los resultados logit muestran que la probabilidad de pasar del empleo formal al autoempleo disminuye a medida que aumenta la diferencia porcentual entre el salario real del sector inicial y el final. La media de la diferencia entre los ingresos reales de la muestra permitió observar que las personas que se desplazaron en ese sentido ganaban menos. Por lo tanto, si esta diferencia aumentara, sería menos probable que los trabajadores pasaran al sector informal.

Del mismo modo, se hizo un seguimiento de las transiciones entre el empleo formal y las dos situaciones en las que no se participa en el mercado de trabajo: el desempleo y fuera de la fuerza de trabajo. En ambos casos, la probabilidad de estas transiciones disminuye a medida que aumenta el nivel de educación. También es importante señalar que la probabilidad de que los trabajadores pasen del sector formal al desempleo disminuye a medida que aumentan los años de experiencia.

En cuanto al segundo análisis logit multinomial, se observa una pauta interesante en los flujos desde el empleo asalariado informal hacia el sector formal. Si tenemos en cuenta que el número de trabajadores con un nivel de educación superior eleva la media de años de instrucción en el sector formal, en el cuadro A1.3 del anexo se muestra que la media de los años de instrucción de las personas que permanecieron como trabajadores asalariados informales era de 5, mientras que la de las personas que pasaron al sector formal era de 6 . Los resultados sugieren que las personas con un mayor nivel de instrucción tienen más probabilidades de ingresar al sector del empleo formal. Esta correlación puede sugerir que los trabajadores comienzan en el sector informal -al considerarlo una opción de empleo mientras prosiguen su educación-y, después de elevar su nivel de instrucción y habilidades, procuran obtener mejores condiciones laborales en el sector formal.

Los resultados también muestran que la educación tiene un efecto positivo en la transición del empleo asalariado informal al autoempleo, en otras palabras, en los movimientos dentro del sector informal. Sin embargo, la diferencia porcentual entre el salario real del sector inicial y el final tiene un impacto negativo sobre este flujo.

En la tercera sección del cuadro A1.3 del anexo se presentan las transiciones desde el autoempleo hacia los otros sectores laborales. La probabilidad de pasar del autoempleo al sector formal se asocia con dos variables: la educación y la diferencia porcentual entre los salarios reales. Con respecto a la primera, las personas con un mayor nivel de educación son más propensas a desplazarse hacia mejores puestos de trabajo en el sector formal. La media de las diferencias entre el salario real de los trabajadores que pasaron al sector formal es positiva y significativa, por lo que, si esa diferencia aumenta, también lo hace la probabilidad de pasar al sector formal.

Los resultados sugieren que las personas con un nivel más alto de educación tienen menos probabilidades de pasar del autoempleo al empleo asalariado informal, lo que sugiere que los trabajadores prefieren la autonomía que este último probablemente no ofrecería. En este caso, la diferencia entre el salario real es positiva.

Por último, en lo que respecta a la transición del sector informal (empleo asalariado y autoempleo) hacia el desempleo o hacia fuera de la fuerza de trabajo, la conclusión principal es que la diferencia porcentual entre el salario real incide de forma negativa sobre esos dos flujos.

Para tener una mejor idea de la importancia económica que tiene el efecto de las características observables de los trabajadores en las diferentes transiciones entre los sectores del mercado de trabajo, se utilizaron los dos modelos logit multinomiales para hacer simulaciones en las que se asignaban determinadas características de los trabajadores ${ }^{5}$. En primer lugar, se analizó el efecto que

\footnotetext{
5 Los resultados de las simulaciones de otras características se omiten para ahorrar espacio, pero la autora los puede proporcionar a solicitud.
} 
tenían los años de experiencia sobre la probabilidad de pasar del sector formal hacia otros sectores y sobre la probabilidad de permanecer en el mismo sector. Para hacer esta estimación, se estableció la edad en 38 años y el nivel de educación en la enseñanza secundaria, y luego se compararon las probabilidades de desplazarse o permanecer, tanto en el caso de los hombres como de las mujeres (gráficos A1.1A a A1.1E del anexo). En ambos sexos, la mayor cantidad de años de experiencia dio lugar a un aumento considerable de la probabilidad de permanecer en el sector del empleo asalariado formal. La probabilidad de las transiciones muestra que las tasas de salida hacia el sector informal y hacia el del desempleo disminuyen a medida que aumentan los años de experiencia.

También se analizó el efecto de los diferentes niveles de educación en el segundo y tercer modelo logit. Una vez más, se estableció la edad en 38 años y se asignaron 10 años de experiencia (véanse los gráficos A1.2A a A1.2I en el anexo). Es interesante observar que, a medida que el nivel de educación aumenta, también lo hace la probabilidad de pasar del sector informal (empleo asalariado y autoempleo) al sector formal. El análisis de la probabilidad de permanecer como trabajador asalariado informal muestra que esta disminuye a medida que aumenta el nivel de educación. Si se utilizan las mismas características de los trabajadores, la probabilidad de pasar del empleo asalariado al autoempleo, al desempleo y a fuera de la fuerza de trabajo aumenta a medida que se incrementa el nivel de educación. Dadas las características mencionadas de los trabajadores, la pauta de la probabilidad de permanecer en el autoempleo es interesante: esta probabilidad aumenta a medida que los trabajadores alcanzan la enseñanza secundaria, y luego disminuye a partir de ahí. Por último, la probabilidad de pasar del autoempleo al empleo asalariado o a fuera de la fuerza de trabajo disminuye a medida que aumenta el nivel de educación.

\section{Observaciones finales}

La informalidad es un fenómeno generalizado que afecta a una proporción considerable de la fuerza de trabajo de muchas economías en desarrollo y en transición. En el estudio se ofrece un panorama general de la dinámica del sector formal y el informal, así como algunas pautas de transición particulares en el interior de los sectores laborales. Se establecieron una matriz de transición y un modelo logit multinomial para averiguar qué movimientos había entre los sectores y qué efecto tenía cada una de las características de los trabajadores sobre la probabilidad de ingresar en un sector o salir de él.

Los resultados que se observaron en la matriz de transición sugieren que hay flujos dinámicos no solo hacia dentro y hacia fuera del mercado laboral, sino también entre los sectores. No obstante, se puede considerar que estos resultados se encuentran dentro del intervalo porcentual normal de los movimientos que se han hallado en análisis similares de la movilidad laboral en América Latina. El desplazamiento de personas ocupadas de un sector a otro sugiere que se buscan oportunidades de empleo en el sector formal y en el informal, como lo ilustran los flujos de la matriz de transición hacia el sector formal, el empleo asalariado informal y el autoempleo.

El análisis logit multinomial se aplicó debido a que en las matrices de transición no se consideran las características observables que pueden afectar la elección de un sector por parte de los trabajadores. Este enfoque indicó que la educación, los años de experiencia y otras características inciden en el tipo de empleo que se selecciona y, por consiguiente, en las transiciones hacia los diversos sectores o la permanencia en ellos.

Los resultados que se presentan en el estudio señalan que hay una interacción importante entre el sector formal y el informal. Las pautas de movilidad sugieren que el empleo informal se debe considerar como un destino deseable, al igual que el sector formal. Las tres situaciones de empleo presentan diversas ventajas y desventajas, según las preferencias y las capacidades individuales. 
Por último, los posibles flujos del mercado laboral desde el empleo formal hacia el empleo asalariado informal y el autoempleo tienen grandes repercusiones en las políticas, ya que estas deberían variar en función de si las personas tienen un empleo formal o uno informal. Por consiguiente, puede resultar útil cuestionar la importancia y la eficacia de las políticas que se aplican en la actualidad al mercado de trabajo, la productividad y la protección social. Si los trabajadores del sector informal se incorporan a él contra su voluntad, los encargados de la formulación de políticas deben centrarse en aspectos como la rigidez salarial y cerciorarse de que las empresas cumplan con sus obligaciones de protección social. Otra cuestión importante es el impacto del empleo informal en la economía: si bien la informalidad es favorable desde el punto de vista personal, desde una perspectiva social es necesario que la economía tenga cierto grado de formalización. En la mayoría de los casos, ingresar al sector informal es la decisión óptima para los trabajadores en función de sus preferencias, nivel de educación, y las restricciones o limitaciones del sector formal. Sin embargo, una fuerza de trabajo informal grande no es necesariamente la mejor opción para la sociedad en su conjunto, ya que la formalización es la manera más fácil de organizar la fuerza de trabajo y el correspondiente marco jurídico.

Cuando ocurre que los trabajadores ingresan de forma voluntaria al sector informal, los encargados de la formulación de políticas deben ser conscientes de la heterogeneidad y la complejidad del empleo y las condiciones de trabajo en el sector informal, y tener en cuenta las ineficiencias de la legislación laboral y el bajo nivel de productividad del sector formal ${ }^{6}$. En este sentido, los trabajadores que se encuentran de forma voluntaria en el sector informal deben encontrar la forma de sustituir la protección que ofrecen las instituciones formales; ello exigiría una evaluación a fondo de la calidad de diversos empleos en lugar de las protecciones jurídicas. Con frecuencia se considera evidente que, dado el bajo nivel de capital humano que hay en el sector informal, mejorar el acceso a la educación y su calidad aumentará la productividad de los trabajadores y la remuneración en el sector formal. Sin embargo, al elegir el sector en que prefieren trabajar, las personas no solo toman en cuenta lo que puedan ganar, sino también todos los demás elementos y ventajas que se asocian con cada puesto de trabajo o sector.

\section{Bibliografía}

Aroca, P. y W. Maloney (1998), "Logit analysis in a rotating panel context and an application to self-employment decisions", Policy Research Working Paper, № 2069, Washington, D.C., Banco Mundial.

Bacheta, M., E. Ernst y J. Bustamante (2009), La globalización y el empleo informal en los países en desarrollo, Ginebra, Organización Internacional del Trabajo (OIT)/Organización Mundial del Comercio (OMC).

Banco Mundial (2012), Ecuador: las caras de la informalidad, Informe № 67808-EC, Washington, D.C.

Bosch, M. y W. Maloney (2005), "Labor market dynamics in developing countries. Comparative analysis using continuous time Markov processes", Policy Research Working Paper, Nㅜ 3583, Washington, D.C.

Canelas, C. (2014), "Minimum wage and informality in Ecuador", Wider Working Paper, № 2014/006, Helsinki, Instituto Mundial de Investigaciones de Economía del Desarrollo (UNU-WIDER).

Cea, S. y M. I. Contreras (2008), "Transiciones laborales: evidencia para datos de panel", tesis, Santiago, Universidad de Chile.

Charmes, J. (2009), "Concepts, measurement and trends", Is Informal Normal? Towards More and Better Jobs in Developing Countries, J. Jütting y J. Laiglesia (eds.), París, Organización de Cooperación y Desarrollo Económicos (OCDE).

De Soto, H. (2000), The Mystery of Capital: Why Capitalism Triumphs in the West and Fails Everywhere Else, Nueva York, Basic Books.

Duryea, S. y otros (2006), "For better or for worse? Job and earnings mobility in nine middle- and low-income countries", Brookings Trade Forum. Global Labor Markets?, Washington, D.C., Brookings Institution Press.

6 Véase Maloney (2004). 
Fields, G. (2009), "Segmented labor market models in developing countries", The Oxford Handbook of Philosophy of Economics, D. Ross y H. Kincaid (eds.), Oxford University Press.

(1972), "Rural-urban migration, urban unemployment and underemployment, and job-search activity in LDC's", Journal of Development Economics, vol. 2, № 2, Amsterdam, Elsevier.

Günther, I. y A. Launov (2006), "Competitive and segmented informal labor markets", IZA Discussion Papers, № 2349, Bonn, Instituto para el Estudio del Trabajo (IZA).

Harris, J. y M. Todaro (1970), "Migration, unemployment and development: a two-sector analysis", American Economic Review, vol. 60, № 1, Nashville, Tennessee, American Economic Association.

Jiménez, M. (2011), "La economía informal y el mercado laboral en la Argentina: un análisis desde la perspectiva del trabajo decente", Documento de Trabajo, № 116, La Plata, Centro de Estudios Distributivos, Laborales y Sociales (CEDLAS).

Jütting, J., J. Parlevliet y T. Xenogiani (2008), "Informal employment re-loaded", IDS Bulletin, vol. 39, № 2, Instituto de Estudios para el Desarrollo.

Lehmann, H. y N. Pignatti (2008), "Informal employment relationships and labor market segmentation in transition economies: evidence from Ukraine", IZA Discussion Paper, №3269, Bonn, Instituto para el Estudio del Trabajo (IZA).

Maloney, W. (2004), "Informality revisited", World Development, vol. 32, № 7, Amsterdam, Elsevier.

_ (1999), "Does informality imply segmentation in urban labor markets? Evidence from sectorial transitions in Mexico", The World Bank Economic Review, vol. 13, № 2, Oxford University Press.

Mandelman, F. y G. Montes-Rojas (2009), "Is self-employment and micro-entrepreneurship a desired outcome?", World Development, vol. 37, №12, Amsterdam, Elsevier.

OIT (Organización Internacional del Trabajo) (2013), La medición de la informalidad: manual estadístico sobre el sector informal y el empleo informal, Ginebra.

Perry, G. y otros (2007), "La razón de ser del sector informal”, Informalidad: escape y exc/usión, Washington, D.C., Banco Mundial.

Slonimczyk, F. y V. Gimpelson (2013), "Informality and mobility: evidence from Russian panel data", IZA Discussion Paper, № 7703, Bonn, Instituto para el Estudio del Trabajo (IZA).

Tansel, A. y E. Oznur (2012), "The formal/informal employment earnings gap: evidence from Turkey", IZA Discussion Paper, № 6556, Bonn, Instituto para el Estudio del Trabajo (IZA). 


\title{
Anexo A1
}

\author{
Cuadro A1.1
}

Ecuador: movilidad de los trabajadores entre los sectores del mercado de trabajo, 2007-2008

(En porcentajes)

\begin{tabular}{lccccccc}
\hline & \multicolumn{7}{c}{ Situación final } \\
\cline { 2 - 8 } Situación inicial & $\begin{array}{c}\text { Empleo } \\
\text { asalariado } \\
\text { formal }\end{array}$ & $\begin{array}{c}\text { Empleo } \\
\text { asalariado } \\
\text { informal }\end{array}$ & $\begin{array}{c}\text { Autoempleo } \\
\text { informal }\end{array}$ & Desempleo & $\begin{array}{c}\text { Fuera de la } \\
\text { fuerza de } \\
\text { trabajo }\end{array}$ & Total & $P_{i}$ \\
\hline Empleo asalariado formal & 79 & 6 & 6 & 2 & 6 & 100 & 27 \\
\hline Empleo asalariado informal & 21 & 46 & 18 & 4 & 12 & 100 & 13 \\
\hline Autoempleo informal & 8 & 7 & 67 & 1 & 16 & 100 & 22 \\
\hline Desempleo & 23 & 11 & 25 & 19 & 22 & 100 & 4 \\
\hline Fuera de la fuerza de trabajo & 4 & 4 & 8 & 3 & 81 & 100 & 34 \\
\hline$P_{j}$ & 26 & 10 & 24 & 3 & 37 & 500 & \\
\hline
\end{tabular}

Fuente: Elaboración propia sobre la base de la Encuesta Nacional de Empleo, Desempleo y Subempleo (ENEMDU) 2011-2012. Nota: $\quad P_{i}$ es el tamaño relativo de un sector en el período inicial; $P_{j}$ es el tamaño relativo de un sector en el período final.

Cuadro A1.2

Ecuador: movilidad de los trabajadores entre los sectores del mercado de trabajo, 2009-2010 (En porcentajes)

\begin{tabular}{lccccccc}
\hline & \multicolumn{7}{c}{ Situación final } \\
\cline { 2 - 7 } Situación inicial & $\begin{array}{c}\text { Empleo } \\
\text { asalariado } \\
\text { formal }\end{array}$ & $\begin{array}{c}\text { Empleo } \\
\text { asalariado } \\
\text { informal }\end{array}$ & $\begin{array}{c}\text { Autoempleo } \\
\text { informal }\end{array}$ & Desempleo & $\begin{array}{c}\text { Fuera de la } \\
\text { fuerza de } \\
\text { trabajo }\end{array}$ & Total & $P_{i}$ \\
\hline Empleo asalariado formal & 75 & 8 & 8 & 2 & 6 & 100 & 25 \\
\hline Empleo asalariado informal & 19 & 47 & 17 & 4 & 13 & 100 & 12 \\
\hline Autoempleo informal & 10 & 9 & 61 & 3 & 18 & 100 & 23 \\
\hline Desempleo & 24 & 14 & 16 & 16 & 30 & 100 & 5 \\
\hline Fuera de la fuerza de trabajo & 5 & 4 & 7 & 3 & 81 & 100 & 35 \\
\hline$P_{i}$ & 27 & 12 & 21 & 4 & 37 & & \\
\hline
\end{tabular}

Fuente: Elaboración propia sobre la base de la Encuesta Nacional de Empleo, Desempleo y Subempleo (ENEMDU) 2011-2012. Nota: $\quad P_{i}$ es el tamaño relativo de un sector en el período inicial; $P_{j}$ es el tamaño relativo de un sector en el período final. 
Cuadro A1.3

Ecuador: estadísticas resumidas en las que se usa la primera definición del sector informal, $2011-2012^{\mathrm{a}}$

\begin{tabular}{|c|c|c|c|c|c|}
\hline \multirow{2}{*}{$\begin{array}{l}\text { Transiciones de los trabajadores } \\
\text { entre los sectores }\end{array}$} & \multicolumn{5}{|c|}{ Media } \\
\hline & $\begin{array}{c}\text { Cantidad de } \\
\text { observaciones }\end{array}$ & $\begin{array}{c}\text { Edad } \\
\text { (en años) }\end{array}$ & $\begin{array}{c}\text { Instrucción } \\
\text { (en años) }\end{array}$ & $\begin{array}{l}\text { Experiencia } \\
\text { (en años) }\end{array}$ & $\begin{array}{l}\text { Salario real inicial } \\
\text { (en dólares) }\end{array}$ \\
\hline \multicolumn{6}{|l|}{ Desde el sector formal hacia } \\
\hline \multirow[t]{2}{*}{ Sector formal } & 1841 & 39,2 & 7,4 & 9,8 & 466,2 \\
\hline & & $(12,91)$ & $(1,93)$ & $(10,38)$ & $(505,70)$ \\
\hline \multirow[t]{2}{*}{ Sector informal (empleo asalariado) } & 171 & 35,6 & 5,9 & 7,3 & 257,4 \\
\hline & & $(12,95)$ & $(1,73)$ & $(9,02)$ & $(221,01)$ \\
\hline \multirow[t]{2}{*}{ Sector informal (autoempleo) } & 178 & 41,6 & 6,8 & 8,1 & 366,9 \\
\hline & & $(12,55)$ & $(1,99)$ & $(8,97)$ & $(392,70)$ \\
\hline \multirow[t]{2}{*}{ Desempleo } & 38 & 29,7 & 7,1 & 3,3 & 338,3 \\
\hline & & $(9,67)$ & $(1,91)$ & $(5,03)$ & $(296,44)$ \\
\hline \multirow[t]{2}{*}{ Fuera de la fuerza de trabajo } & 156 & 40,9 & 6,7 & 10,7 & 375,1 \\
\hline & & $(20,14)$ & $(2,02)$ & $(13,65)$ & $(466,04)$ \\
\hline \multicolumn{6}{|c|}{ Desde el sector informal (empleo asalariado) hacia } \\
\hline \multirow[t]{2}{*}{ Sector formal } & 196 & 34,0 & 6,1 & 6,2 & 209,5 \\
\hline & & $(12,69)$ & $(1,73)$ & $(8,19)$ & $(114,56)$ \\
\hline \multirow[t]{2}{*}{ Sector informal (empleo asalariado) } & 438 & 37,3 & 5,2 & 8,66 & 192,6 \\
\hline & & $(14,30)$ & $(1,61)$ & $(10,34)$ & $(81,24)$ \\
\hline \multirow[t]{2}{*}{ Sector informal (autoempleo) } & 190 & 42,4 & 5,3 & 10,5 & 180,1 \\
\hline & & $(12,50)$ & $(1,76)$ & $(12,31)$ & $(98,84)$ \\
\hline \multirow[t]{2}{*}{ Desempleo } & 38 & 32,2 & 5,9 & 7,6 & 178,2 \\
\hline & & $(12,69)$ & $(1,66)$ & $(12,22)$ & $(96,84)$ \\
\hline \multirow[t]{2}{*}{ Fuera de la fuerza de trabajo } & 106 & 36,4 & 5,5 & 6,8 & 129,5 \\
\hline & & $(17,52)$ & $(1,93)$ & $(12,10)$ & $(78,03)$ \\
\hline \multicolumn{6}{|c|}{ Desde el sector informal (autoempleo) hacia } \\
\hline \multirow[t]{2}{*}{ Sector formal } & 200 & 42,6 & 6,8 & 11,3 & 290,8 \\
\hline & & $(14,06)$ & $(1,97)$ & $(11,08)$ & $(394,28)$ \\
\hline \multirow[t]{2}{*}{ Sector informal (empleo asalariado) } & 191 & 42,1 & 5,32 & 11,4 & 180,7 \\
\hline & & $(14,94)$ & $(1,86)$ & $(12,91)$ & $(324,39)$ \\
\hline \multirow[t]{2}{*}{ Sector informal (autoempleo) } & 1591 & 48,0 & 5,5 & 13,6 & 215,0 \\
\hline & & $(13,53)$ & $(1,90)$ & $(12,03)$ & $(302,08)$ \\
\hline \multirow[t]{2}{*}{ Desempleo } & 34 & 30,4 & 7,2 & 4,0 & 107,9 \\
\hline & & $(10,62)$ & $(1,65)$ & $(4,12)$ & $(164,50)$ \\
\hline \multirow[t]{2}{*}{ Fuera de la fuerza de trabajo } & 350 & 48,0 & 5,4 & 10,7 & 90,7 \\
\hline & & $(19,53)$ & $(1,90)$ & $(13,73)$ & $(137,73)$ \\
\hline
\end{tabular}

Fuente: Elaboración propia sobre la base de la Encuesta Nacional de Empleo, Desempleo y Subempleo (ENEMDU) 2011-2012.

a Esta definición del sector informal se refiere a trabajadores asalariados empleados en pequeños establecimientos no registrados que tienen menos de diez empleados, más todos los trabajadores independientes y los que trabajan por cuenta propia. Los errores estándar aparecen entre paréntesis. 
Gráfico A1.1

Ecuador: efecto de los años de experiencia sobre diversas transiciones del mercado de trabajo, por sexo ${ }^{a}$

(Probabilidades)

A. Permanencia en el empleo asalariado formal

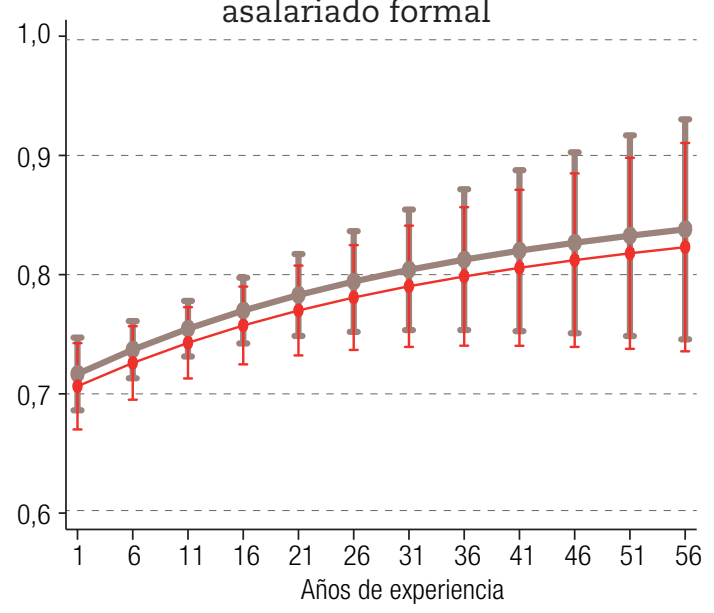

C. Del empleo asalariado formal al autoempleo

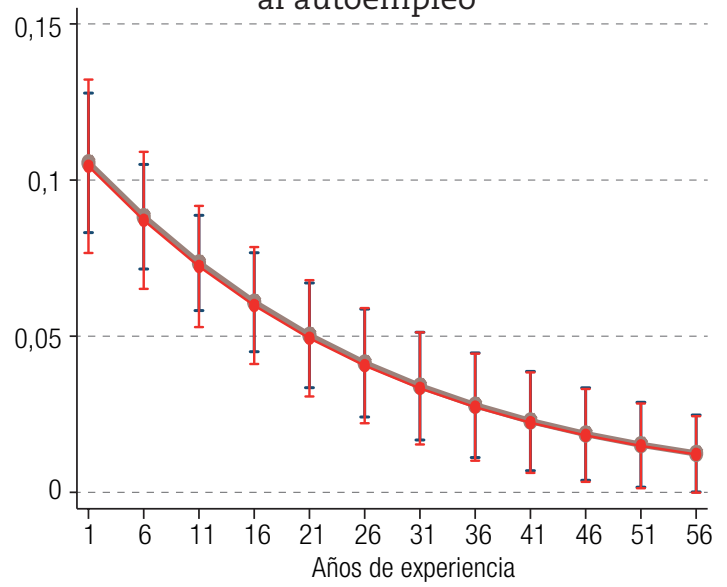

B. Del empleo asalariado formal al empleo asalariado informal

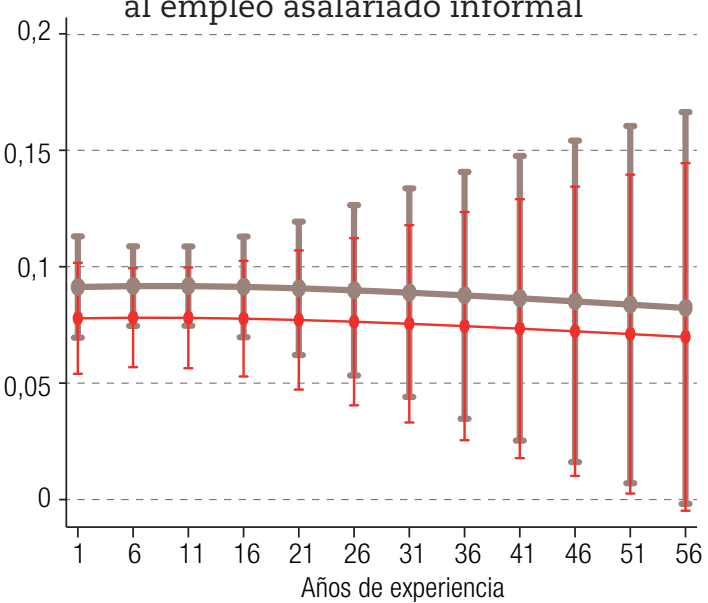

D. Del empleo asalariado formal al desempleo

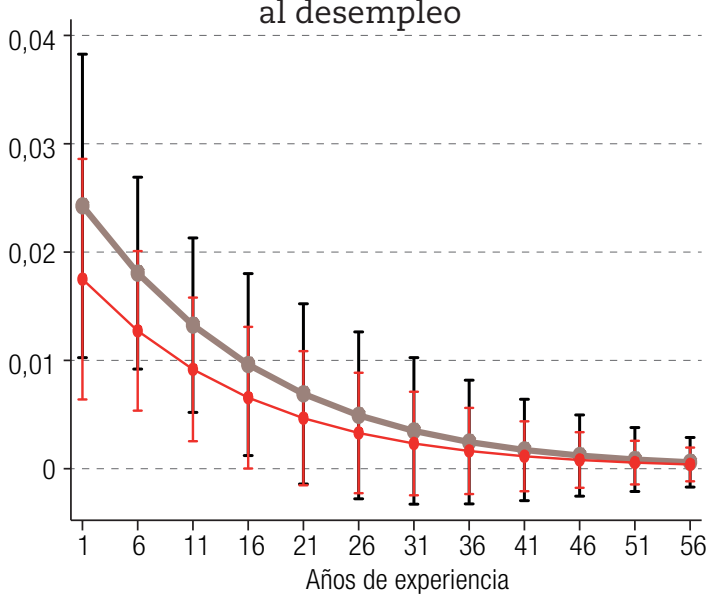

E. Del empleo asalariado formal a fuera de la fuerza de trabajo

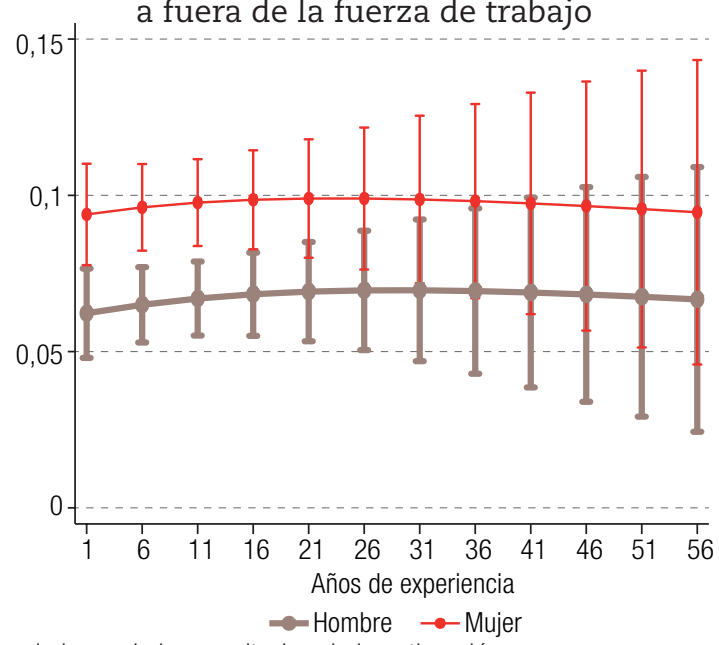

Fuente: Elaboración propia sobre la base de los resultados de la estimación.

a Márgenes de predicción con intervalos de confianza del 95\%. 


\section{Gráfico A1.2}

Ecuador: efecto del nivel de educación sobre diversas transiciones del mercado de trabajo, por sexo ${ }^{\mathrm{a}}$

(Probabilidades)

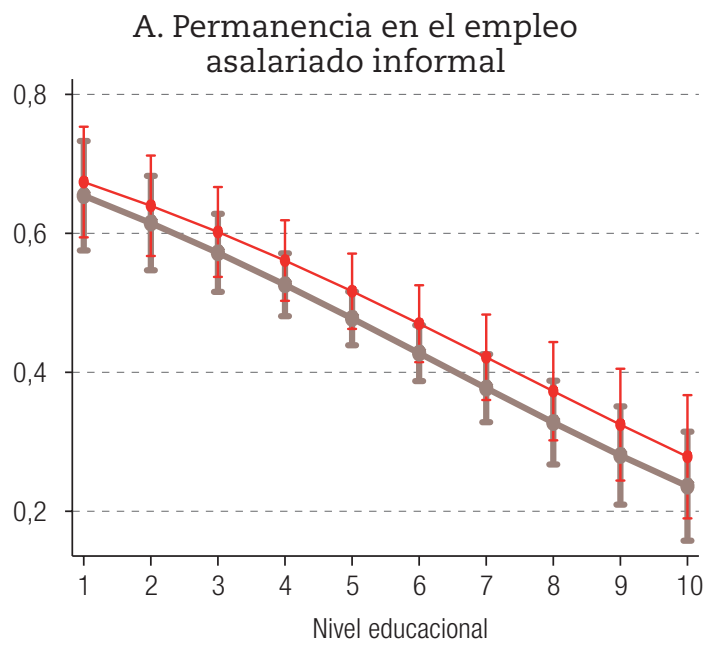

C. Del empleo asalariado informal al autoempleo

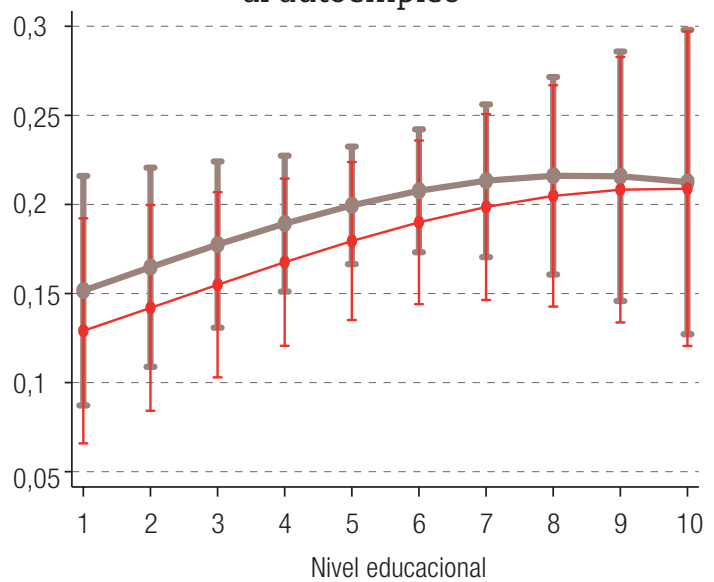

E. Del empleo asalariado informal a fuera de la fuerza de trabajo

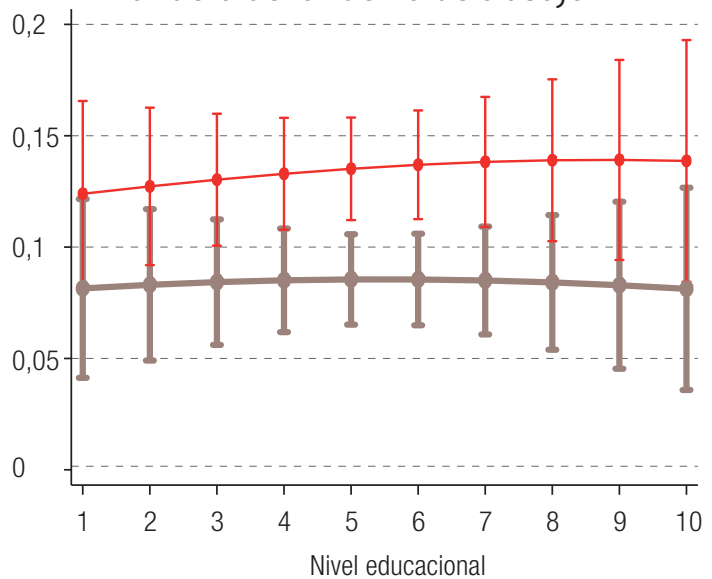

B. Del empleo asalariado informal al empleo asalariado formal

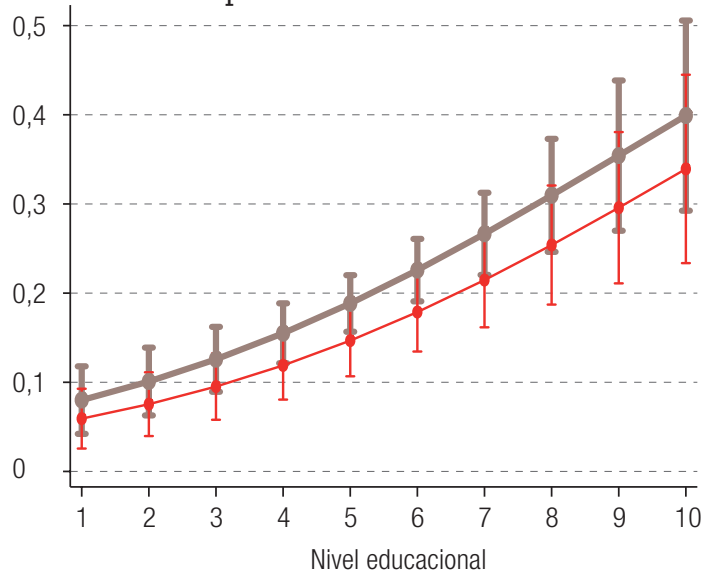

D. Del empleo asalariado informal al desempleo

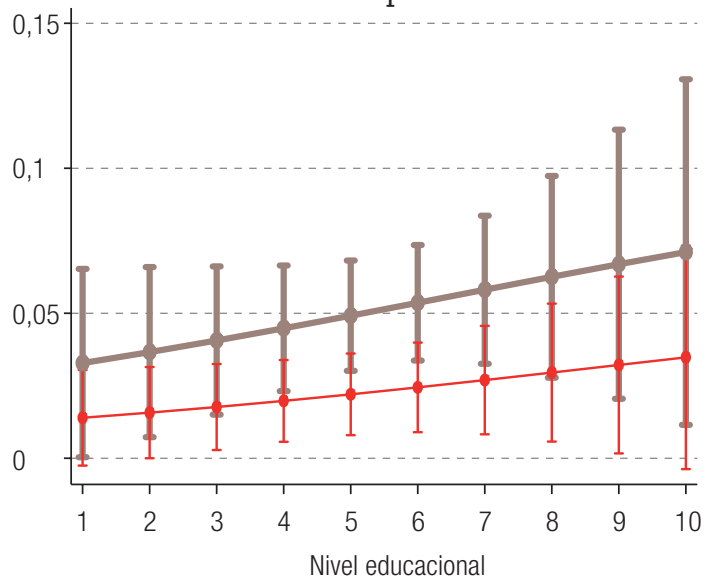

F. Del autoempleo al empleo asalariado formal

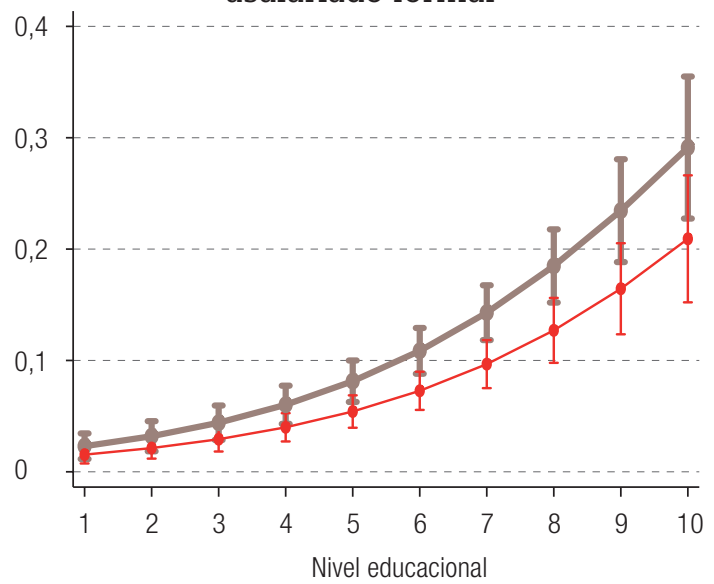


Gráfico A1.2 (conclusión)

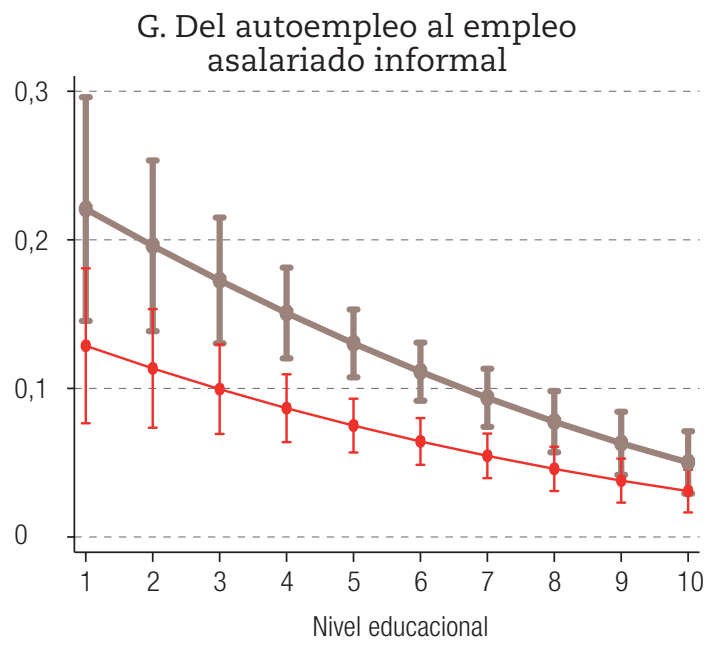

H. Permanencia en el autoempleo

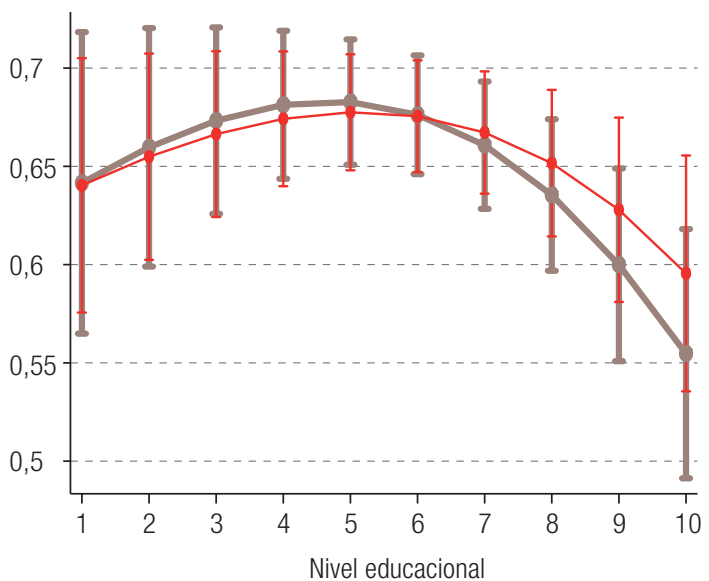

I. Del autoempleo al desempleo
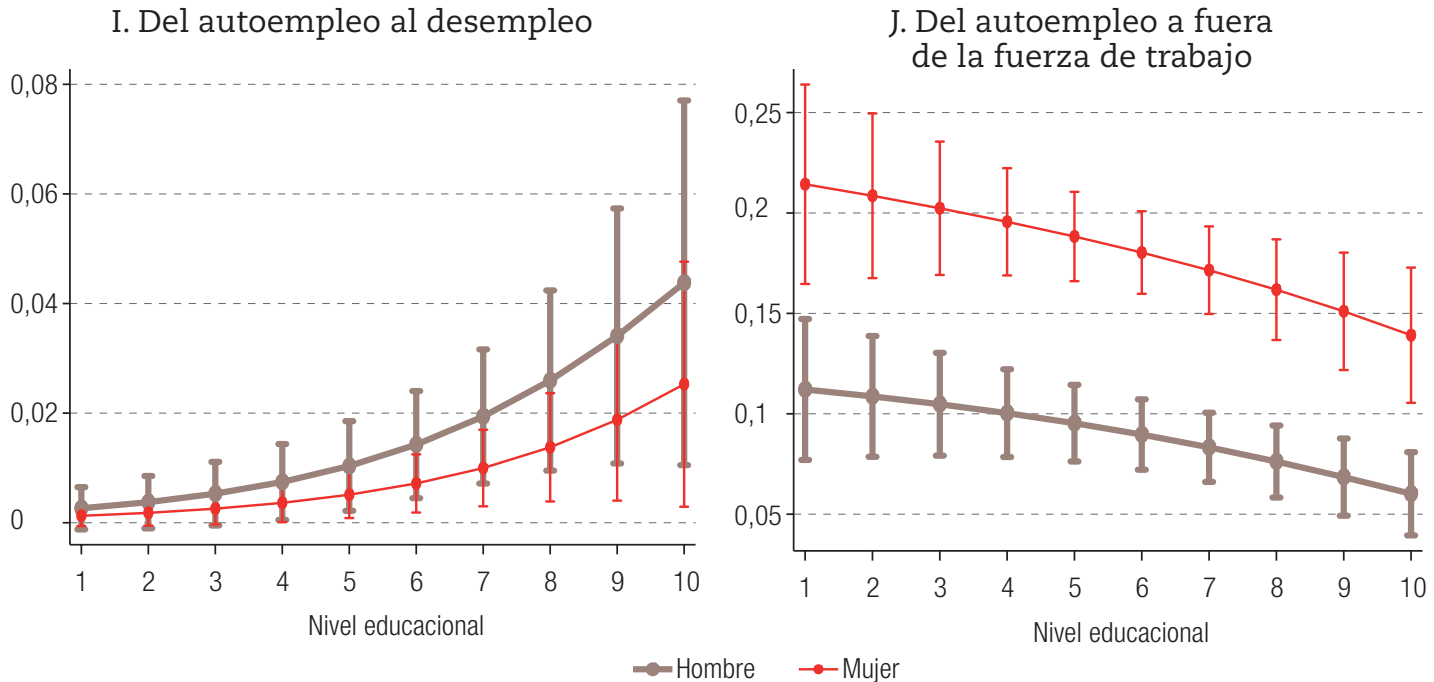

Fuente: Elaboración propia sobre la base de los resultados de la estimación.

a Márgenes de predicción con intervalos de confianza del 95\%. 